\title{
Compulsion in the community: mental health professionals' views and experiences of CTOs
}

\author{
Delphine Coyle, ${ }^{1}$ Rob Macpherson, ${ }^{2}$ Chris Foy, ${ }^{3}$ Andrew Molodynski, ${ }^{4}$ Maya Biju, ${ }^{5}$ Joseph Hayes ${ }^{6}$
}

The Psychiatrist (2013), 37, 315-321, doi: 10.1192/pb.bp.112.038703

${ }^{1}$ Tavistock Centre, London; ${ }^{2}$ Lexham Lodge, Cheltenham; ${ }^{3}$ Gloucestershire Royal Hospital, Gloucester; ${ }^{4}$ University of Oxford, Warneford Hospital, Oxford; ${ }^{5}$ Evergreen House, Cheltenham;

${ }^{6}$ University College London

Correspondence to Delphine Coyle (dcoyle@tavi-port.nhs.uk)

First received 22 Jan 2012, final revision 30 Dec 2012, accepted 15 Jan 2013

\begin{abstract}
Aims and method We surveyed the views and experiences of all mental health professionals in adult community mental health teams and approved mental health professionals in 2 Gether and Oxford Health NHS Foundation Trusts, regarding the use of community treatment orders (CTOs).
\end{abstract}

Results A total of 288 surveys were completed (response rate $48 \%$ ). Forty-eight (83\%) psychiatrists and 142 (67\%) non-psychiatrist mental health professionals were in favour of CTOs. The decision-making regarding CTOs was overwhelmingly clinically oriented for all professional groups. However, there were significant differences in views between groups regarding the effects of bureaucracy, the infringement of human rights and coercion.

\begin{abstract}
Clinical implications Multidisciplinary team involvement is crucial in decisions regarding CTOs and may protect against idiosyncratic or unhelpful practice. Further training for staff is urgently required and there may be a case for creating small local reference groups that can develop expertise and provide advice and support for clinical teams.
\end{abstract}

\section{Declaration of interest None.}

The proposed introduction of community treatment orders (CTOs) in the 1990s in England and Wales brought together opposition from a Mental Health Alliance including the Royal College of Psychiatrists, mental health charities, various groups of professionals, users of services and carers. However, CTOs were eventually introduced in 2007 as a part of the substantially amended Mental Health Act 1983. Their use has considerably exceeded predictions, with over 10000 orders being made in England alone by March 2011. ${ }^{1}$ Formal powers of compulsion in the community have existed for some time in Australasia and North America and have more recently been made available in a number of European countries (including Scotland, Sweden, Belgium, Portugal and Luxemburg). ${ }^{2,3}$ In England and Wales, CTOs do not allow for the forcible administration of medication in the community. A refusing patient can be compelled to return to hospital, at which point they can be forcibly treated if this is felt to be appropriate, and in practice under such circumstances they may decide to accept treatment in the community if they feel under pressure. It is possible for the treating team to make other conditions such as specifying the patient's residency and abstinence from drugs or alcohol. Enforcement of these conditions may however be difficult to achieve in practice. The use of CTOs varies substantially between and within jurisdictions. ${ }^{1}$ Dawson ${ }^{4}$ has suggested that four main factors typically influence clinicians: (a) the legal structure of the CTO regime, (b) the community mental health services available, (c) the clinician's views about the possible impact of coercion on their relations with patients, and (d) the expectations of third parties regarding the CTO scheme.

The evidence of benefits from CTOs is at best limited: two randomised controlled trials (RCTs) of CTOs in the USA showed no overall difference between those on a CTO and those not in terms of readmission and clinical and social outcomes. ${ }^{5,6}$ A Cochrane review concluded that 'there is no strong evidence to support the claims made for compulsory community treatment'. ${ }^{7}$ It has been suggested that a lack of research evidence or clinical consensus regarding CTOs may have contributed to their wider than forecasted uptake. ${ }^{8-10}$ It appears that this relates to a number of factors as outlined by Dawson. ${ }^{4}$ The legislation is enabling and there are broad criteria for its use. Given this, it is likely that factors such as family concerns, defensive practice and a natural clinical desire to alter previously unsuccessful treatment approaches all contribute. Politicians and legislators may also look to out-patient commitment to address a variety of issues in the community management of those with severe mental illnesses such as perceived dangerousness. ${ }^{8,9}$ An RCT of compulsory outpatient treatment in psychosis was conducted in the Oxford Department of Social Psychiatry under Professor Tom Burns and the study seeks to provide convincing scientific evidence of CTO efficacy with the results expected to be published this year. ${ }^{11}$ A national survey of members of the Royal College of Psychiatrists was conducted by Manning 
et al in 2009 to determine the views and experiences of psychiatrists in England and Wales regarding CTOs. ${ }^{12}$ Manning et al noted that a number of authors had examined professional attitudes to this newly developed, complex and politically sensitive legal process. A survey of consultant psychiatrists in England and Wales in 2000 revealed that $46 \%$ were in favour of their introduction, 35\% against and $19 \%$ unsure. $^{13}$ In New Zealand (where CTOs have been available since 1992), surveys of psychiatrists and other mental health professionals found that $79 \%$ of psychiatrists and $85 \%$ of non-psychiatrist mental health professionals preferred to work in a system with CTOs. ${ }^{14}$ A survey of 50 psychiatrists in Saskatchewan, Canada, reported that $62 \%$ of respondents were at least satisfied with the effect of CTOs on patient care. ${ }^{15}$ Two studies in the USA exploring clinicians' views revealed similar opinions. ${ }^{16,17}$ In Manning et al's 2009 survey of members of the Royal College of Psychiatrists, 566 usable questionnaires were returned out of the 1928 questionnaires that were posted out. They found that psychiatrists were generally positive about the introduction of CTOs and $60 \%$ expressed a preference to work in a system that included CTOs. Moreover, clinical reasons were rated as being the most important factors in the use of CTOs, both in terms of commencing and ending orders, rather than ethical or bureaucratic concerns. Promoting adherence to medication, protecting individuals from the consequences of relapse and ensuring contact with health professionals were considered to be the most important factors in commencing a CTO. The most important reasons for discharging a CTO were considered to be the development of insight, clinical improvement and adherence to treatment. ${ }^{12}$

In discussing their findings, Manning et al refer to the importance of seeking multidisciplinary input when making CTO decisions, and note that lack of professional consensus may be a challenging problem. ${ }^{12}$ This study develops the work of Manning et al by using a similar method to explore the views and experiences of a wider range of mental health professionals in a local area. The professionals surveyed included psychiatrists, nurses, social workers, team managers and other occupational groups (including psychologists, support workers, occupational therapists and physiotherapists) and the opinions of the different professional groups were compared and contrasted. We aimed to determine their views regarding the use of CTOs in adult mental health services.

\section{Method}

All medical and non-medical staff in adult community mental health teams in 2 Gether and Oxford Health NHS Foundation Trusts were invited to complete the survey. Researchers attended team meetings to explain the study and hand out questionnaires. Staff were sent a further questionnaire approximately 2 months later to increase the response rate. The approved mental health professional (AMHP) offices in the two trusts provided a comprehensive list of the AMHPs and all were invited to complete the survey. All responses were anonymous.

The study was approved by Gloucestershire 2Gether NHS Foundation Trust and Oxfordshire and Buckinghamshire
Mental Health NHS Foundation Trust Research and Development Governance Committees. The National Research Ethics Service (NRES) advised that the study did not require ethical review by an NHS research ethics committee.

\section{The questionnaire}

The questionnaire was developed to capture views and experiences across a range of issues in relation to the use of CTOs:

- indications for the use of CTOs

- factors influencing how CTOs work

- potential benefits and disadvantages

- potential impact on the therapeutic relationship

- the individual's overall level of support for the introduction of CTOs

In order to allow comparison between this questionnaire and a tool used in previous work on attitudes towards CTOs, ${ }^{12}$ some questions were purposefully replicated. However, this study develops previous work by providing a detailed breakdown of results according to the mental health professional groups. In addition, this questionnaire contains other unique questions designed to further explore the views and experiences of the different professional groups.

\section{Statistical analysis}

Statistical analysis was carried out using SPSS version 18 for Windows. Kruskal-Wallis tests were used to assess differences between the views and experiences of mental health professionals working in different disciplines. $P$-values less than 0.05 were considered to be statistically significant.

\section{Results}

The overall response rate was $48 \%, 288$ of 606 surveys being returned, and $62 \%$ (177) of respondents were female. The majority of respondents were aged between 31 and 50 years (63\%). The ethnic diversity of the sample approximately reflected that of the general population of the $\mathrm{UK}:{ }^{18} 80 \%$ $(n=229)$ of respondents were White British. The majority of respondents $(72 \%)$ worked full time and the mean length of service was 15 years.

A total of $21 \%(n=59)$ of respondents were doctors (of whom $51 \%$ were consultant psychiatrists), $7 \%$ were team managers, $33 \%$ nurses, $18 \%$ social workers and $21 \%$ were classed as 'other occupational group'. This latter group included psychologists, support workers, occupational therapists and physiotherapists. Respondents worked overwhelmingly in community services of different types: $47 \%$ in community mental health teams, $19 \%$ in crisis teams, $18 \%$ in assertive outreach, $12 \%$ in early intervention and $13 \%$ in other teams. The other teams included: emergency duty team/emergency out-of-hours AMHPs, community rehabilitation and criminal justice.

Respondents rated the importance of 12 factors when making the decision to use a CTO on a five-point Likert scale, ranging from one (very important) to five (not 
important at all). The results for the psychiatrists and other mental health professionals in our sample, together with a comparison of factor rankings with UK psychiatrists, ${ }^{12}$ New Zealand psychiatrists ${ }^{14}$ and other mental health professionals, ${ }^{14}$ are shown in Table 1. 'Ensuring contact with mental health professionals' was considered to be the most important factor by four of the five groups. All professional groups agreed on the five most important factors in decision-making.

Respondents rated the importance of nine possible mechanisms influencing how CTOs work. The results are shown in Table 2. The different groups ranked the three most important mechanisms similarly, although there was some difference in emphasis of the importance of adherence with prescribed medication.

Respondents rated nine possible factors that could potentially undermine the effectiveness of CTOs. Again, there was broad agreement, with the only significant difference being the importance ascribed to 'inadequate access to psychological therapies'. Nurses and the 'other' occupational group tended to rate this as greatly undermining the effectiveness of CTOs, whereas it was rated as a relatively unimportant factor by the three other groups.

Respondents rated eight possible factors that could discourage the use of CTOs. Whereas respondents tended to avoid strong opinions, there were several significantly different results. Psychiatrists were more concerned than social workers by the additional administrative burdens. Nurses were more concerned than psychiatrists and social workers by the degree of coercion involved and the nurses were most concerned by the difficulty in accessing secondopinion doctors. Although not a significantly different result, there was a trend for the team managers to be least concerned by the loss of civil liberties.

Table 3 shows how respondents rated their level of agreement with the following statements on a five-point Likert scale: 'I support the use of CTOs' and 'In the use of CTOs, their benefits outweigh any coercive impact on the patient'. The results showed that the vast majority supported the use of CTOs, with $83 \% \quad(n=48)$ of psychiatrists and $67 \%(n=142)$ of non-psychiatrist mental health professionals in support of the use of CTOs. Overall, $74 \%$ of psychiatrists and $50 \%$ of other mental health professionals agreed that the benefits of the use of CTOs outweighed any coercive impact on the patient.

Of the psychiatrists, $52 \%$ believed that CTOs helped the development or maintenance of the therapeutic relationship with the patient and $17 \%$ believed that they hindered the relationship. Those from other professional groups had a less positive view, $45 \%$ believing they helped the relationship and $21 \%$ believing they hindered it.

Respondents rated their level of agreement with seven general statements regarding CTOs, as shown in Table 4. For the statement: 'The introduction of CTOs has been a retrograde step for mental health services', team managers and psychiatrists disagreed with this statement significantly more than the other professions. The same order of agreement was found for the statement: 'CTOs infringe patients' human rights'. All professional groups believed that CTOs provided greater treatment options. Overall, staff of all disciplines reported that the benefits of CTOs could 'already be seen'.

There was overall agreement between the professional groups on a number of general statements regarding CTOs. Respondents disagreed with the proposition that the introduction of CTOs was a 'retrograde step'. All groups believed they provided greater treatment options and could provide additional benefits over and above well-resourced voluntary community services.

\section{Discussion}

The results of this survey of mental health professionals working in England shortly after the introduction of CTOs demonstrate broad agreement across professions on the main issues regarding CTOs. There was also some level of consensus across time and across countries as demonstrated by a comparison of our results with previous UK studies ${ }^{12,13,19}$ and the New Zealand studies. ${ }^{14}$ Psychiatrists and other mental health professionals in England and in New Zealand believed that the same five factors were most important in their decision-making regarding CTOs. These factors were overwhelmingly clinical and included: ensuring contact with professionals, adherence with medication and rapid identification of relapse. The different groups reported similar views on the most important mechanisms influencing how CTOs work, which included ensuring adherence to medication for a lengthy period, ensuring a greater period of stability and signalling to the patient that they have a serious mental problem.

Although a response rate of $48 \%$ is high for this form of research and a strength of our study, respondents were all located in the South of England. It is possible that this may affect the generalisability of the results but it seems reasonable to assume that views are broadly representative of the national picture.

Despite substantial areas of agreement there were some significant differences between the professional groups. Nurses were most likely to feel that inadequate access to psychological therapies could undermine the effectiveness of CTOs and were most concerned by the negative effects of coercion. Psychiatrists (perhaps unsurprisingly, as they are responsible for so much of this) were most concerned regarding the burden of bureaucracy. Our results suggested that professional differences about different aspects of the deployment of CTOs can be expressed within a generally facilitating framework, in which most staff regard CTOs as a potentially valuable process for some cases. These differences could be important in shaping practice and providing balance in decision-making. They may also, if they can be shared in a supportive manner, serve to develop more consistent practice and reduce the considerable variations in practice that can be seen today.

Our respondents believed that CTOs could improve the development of a therapeutic relationship with the patient over time with continued treatment, support and greater insight on the part of the patient. The majority ( $74 \%$ of psychiatrists and $50 \%$ of other mental health professionals) also agreed to some extent that the benefits of CTOs outweighed any coercive impact on the patient. The vast majority supported the use of CTOs, with $83 \%$ of psychiatrists 


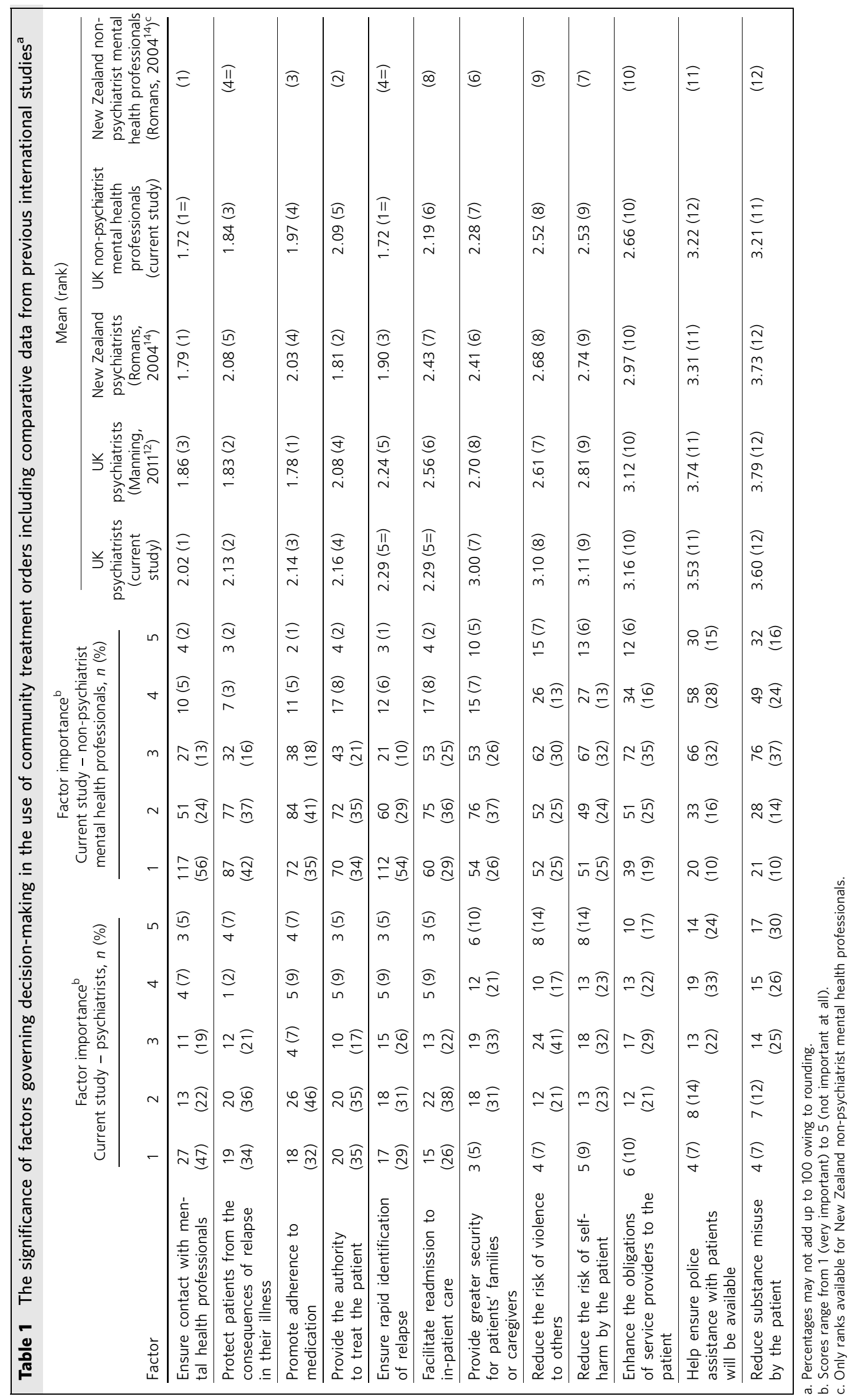



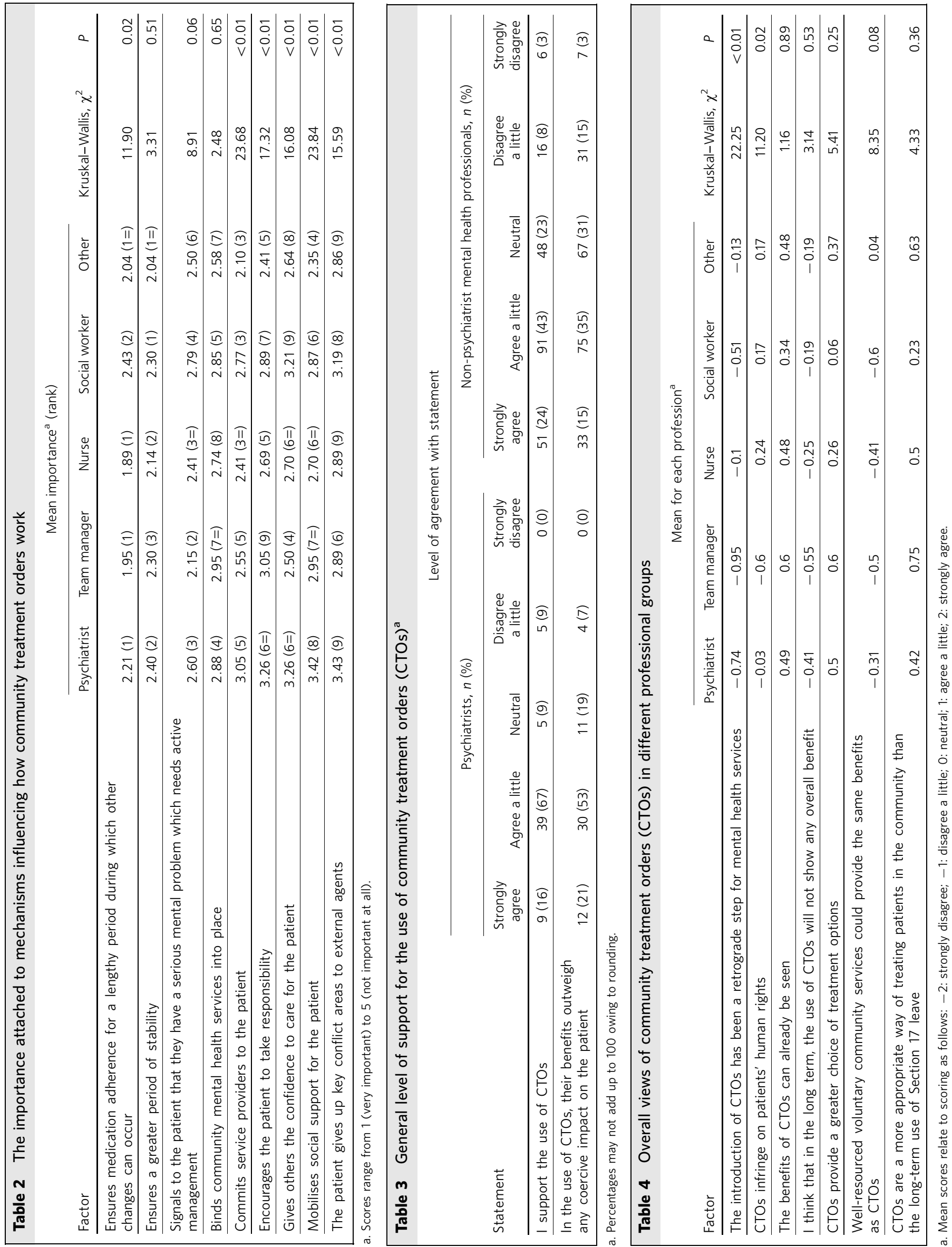
and $67 \%$ of other professionals in favour of their continued availability. It would appear that there has been a marked shift in the mental health professionals' views and experiences of CTOs since the New Zealand mental health professionals' survey ${ }^{14}$ and the UK psychiatrists' survey. ${ }^{12}$ The New Zealand surveys of consultant psychiatrists and other mental health professionals found that $79 \%$ of psychiatrists and $85 \%$ of non-psychiatrist mental health professionals preferred to work in a system with CTOs. ${ }^{14}$ The survey of UK psychiatrists in 2009 found that $60 \%$ expressed a preference for working in a system with CTOs ${ }^{12}$ and our figure of $83 \%$ suggests a substantial increase in the level of support for the use of CTOs among psychiatrists in the few years since CTOs have been in use.

Although the introduction of CTOs in England was combined with a national training programme for psychiatrists and AMHPs, many from other professional backgrounds have had little or no training in this area. We believe that this is a concern as in many cases community psychiatric nurses and occupational therapists are the main professionals working with people subject to CTOs. Our survey demonstrates substantial experience and considerable understanding among such staff. The decision to initiate a CTO should be a multidisciplinary one and the differences in outlook suggested by our survey may protect against idiosyncratic or unhelpful practice. However, if this is to be the case, more education and training needs to be made available to staff of all professional groups who are involved in decisions regarding CTOs. This would enable other professionals to take a full role in decisions about deployment, ongoing management and discharge of CTOs - discharge being relatively unusual in practice so far. ${ }^{1}$

We believe that it may be valuable to develop small groups in an area that can develop expertise and acquire more detailed knowledge regarding clinical practice involving compulsion. Such a group may include clinicians from different disciplines alongside service users and carers to provide a broad-based approach to such decisions. They could act as a resource for clinical teams who might only face these decisions relatively infrequently. Such a body may function in a similar way to an accommodation panel or a hospital ethics group by being available to consider cases with clinical teams on a fairly informal basis and provide advice and support. The question of resources inevitably arises but the benefits of a more coordinated and considered approach to the use of compulsion in community care could outweigh the resource burden.

\section{Further research}

The benefits of CTOs remain uncertain and there is a lack of high-quality research evidence for the outcomes and effectiveness of CTOs. Only two RCTs have been conducted, both in the USA. ${ }^{5,6}$ Neither show an overall difference in outcome between those on a CTO and those not in terms of readmission and clinical and broadly defined social functioning. Also, CTOs have the potential to infringe on human rights such as Article 3 (prohibition of torture, which includes inhuman or degrading treatment), Article 5 (right to liberty and security) and Article 8 (right to respect for private and family life) of the Human Rights Act $1998 .^{20}$ Given this, there remain concerns regarding the practice of restricting patients' freedoms under a CTO when there is little objective evidence of improved outcome as a result of such restrictions. There is a lack of professional consensus or guidance in this area of practice. Ultimately, the current lack of evidence needs to be addressed and further research is urgently required, including studies that consider the experiences and views of service users and carers and to analyse the processes involved in deciding on and utilising compulsion.

\section{Funding}

The 2Gether NHS Foundation Trust Research and Development fund was used to cover the relatively small costs of the study.

\section{Acknowledgements}

We thank Genevieve Riley, Krishen Ranganath, Mark Walker, Caroline Marrow and Nicola Hovey for their help with the design and implementation of the study and Cate Manning for allowing the use of part of her research instrument. We also thank Mike Blackburn, Caroline Jennings, Anne Jones, Judith Rimell and Dave Buckle of the Gloucester assertive outreach team for their positive involvement, and all other staff who participated.

\section{About the authors}

Delphine Coyle is a specialty trainee (year 5) psychiatrist at the Tavistock and Portman NHS Foundation Trust, London. Rob Macpherson is a consultant psychiatrist at Gloucestershire 2Gether NHS Foundation Trust, Cheltenham. Chris Foy is a medical statistician at the Gloucestershire Research Support Service, Gloucestershire Royal Hospital, Gloucester. Andrew Molodynski is a consultant psychiatrist at the Oxford Health NHS Foundation Trust, Oxford. Maya Biju is a core trainee (year 2) psychiatrist at Gloucestershire 2Gether NHS Foundation Trust, Cheltenham. Joseph Hayes is a clinical training fellow in the Mental Health Sciences Unit at University College London.

\section{References}

1 NHS Information Centre. Inpatients Formally Detained in Hospitals under the Mental Health Act 1983 and Patients Subject to Supervised Community Treatment, Annual Figures, England 2010/11. The Health and Social Care Information Centre, 2011

2 Salize HJ, Dreßing H, Peitz M. Compulsory Admission and Involuntary Treatment of Mentally III Patients - Legislation and Practice in European Union Member States. European Commission Health \& Consumer Protection Directorate, 2002

3 Lawton-Smith S. Community-based Compulsory Treatment Orders in Scotland. King's Fund, 2006.

4 Dawson J. Factors influencing the rate of use of community treatment orders. Psychiatry 2007; 6: 42-4

5 Swartz MS, Swanson JW, Wagner HR, Burns BJ, Hiday VA, Borum R. Can involuntary outpatient commitment reduce hospital recidivism? Findings from a randomised trial with severely mentally ill individuals. Am J Psychiatry 1999; 156: 1968-75.

6 Steadman HJ, Gounis K, Dennis D, Hopper K, Roche B, Swartz M, et al. Assessing the New York City involuntary outpatient commitment pilot program. Psychiatr Serv 2001; 52: 330-6.

7 Kisely S, Campbell LA, Preston N. Compulsory community and involuntary outpatient treatment for people with severe mental disorders. Cochrane Database Syst Rev 2005; 3: CD004408.

8 Appelbaum PS. Thinking carefully about outpatient commitment. Psychiatr Serv 2001; 52: 347-50. 
9 Monahan J, Bonnie RJ, Appelbaum PS, Hyde PS, Steadman HJ, Swartz MS. Mandated community treatment: beyond outpatient commitment. Psychiatr Serv 2001; 52: 1198-205.

10 Dawson J, Romans S, Gibbs A, Ratter N. Ambivalence about community treatment orders. Int J Law Psychiatry 2003; 26: 243-55.

11 Burns T, Rugkåsa J, Dawson J, Doll H, Molodynski A, Priebe S, et al. 10PRT/0496: Oxford Community Treatment Order Evaluation Trial (OCTET): A Single-Outcome Randomised Controlled Trial of Compulsory Outpatient Treatment in Psychosis (ISRCTN73110773). The Lancet, 2010 (http://www.thelancet.com/protocol-reviews/10PRT-0496).

12 Manning C, Molodynski A, Rugkåsa J, Dawson J, Burns T. Community treatment orders in England and Wales: national survey of clinicians' views and use. Psychiatrist 2011; 35: 328-33.

13 Crawford MJ, Hopkins W, Henderson C. Concerns over reform of the Mental Health Act [letter]. Br J Psychiatry 2000; 177: 563.

14 Romans S, Dawson J, Mullen R, Gibbs A. How mental health clinicians view community treatment orders: a national New Zealand survey. Aust N Z J Psychiatry 2004; 38: 836-41.
15 O'Reilly RL, Keegan DL, Elias JW. A survey of the use of community treatment orders by psychiatrists in Saskatchewan. Can J Psychiatry 2000; 45: 79-81.

16 Scheid-Cook TL. Controllers and controlled: an analysis of participant constructions of outpatient commitment. Soc Health IIIness 1993; 15: 179-98.

17 Swartz MS, Swanson JW, Wagner HR, Hannon MJ, Burns BJ, Shumway M. Assessment of four stakeholder groups' preferences concerning outpatient commitment for persons with schizophrenia. Am J Psychiatry 2003; 160: 1139-46.

18 Office for National Statistics. United Kingdom Population by Ethnic Group. United Kingdom Census 2011. ONS, 2011 (http://www.ons.gov.uk/ons/ guide-method/census/2011/index.html).

19 Bindman J. Involuntary outpatient treatment in England and Wales. Curr Opin Psychiatry 2002; 15: 595-8.

20 Council of Europe. Convention for the Protection of Human Rights and Fundamental Freedoms (European Convention on Human Rights, as amended) (ECHR). Council of Europe, 1950. 